\title{
Prevalence of hearing impairment in patients with diabetes mellitus at tertiary care center of Nepal
}

\begin{abstract}
Background: Diabetes mellitus is a chronic, systemic and metabolic disorder known to affect almost every organ due to its unequivocal complications. The auditory organ is often affected but usually ignored complications of diabetes mellitus. With the rise in the prevalence of diabetes mellitus and hearing impairment in developing countries, it becomes a crucial public health issue if both of them are found to be associated.
\end{abstract}

Objective: To find the prevalence of hearing impairment in diabetic patients.

Materials and methods: This was a cross-sectional study conducted at the Department of Clinical Physiology in collaboration with the Department of General Practice and Emergency Medicine and the Department of Otorhinolaryngology- Head and Neck Surgery, Tribhuvan University Teaching Hospital. After applying exclusion criteria, 55 subjects were recruited for the study. Pure Tone Audiometry, a non-invasive test was used to assess hearing threshold in this study. Audiogram findings of diabetics were then analyzed.

Results: Outcomes of the tests revealed that $17(30 \%)$ of subjects with diabetes mellitus had hearing loss, out of which 13 had mild hearing loss. A significantly positive correlation was found between age and hearing threshold at every frequency. Higher frequencies were found to be more affected. The best cut-off age for onset of hearing loss in diabetics was found to be 51.50 years.

Conclusion: Current study suggests a 30\% prevalence of hearing loss in diabetes mellitus. We observed hearing loss more among the elderly at higher frequencies.

Keywords: audiometry, diabetes mellitus, hearing loss
Volume 8 Issue 2 - 202 I

\author{
Neeti Bhat,' Narayan Bahadur Mahotra,' \\ Lava Shrestha,' Tirtha Man Shrestha, ${ }^{2}$ \\ Prashant Tripathi, ${ }^{3}$ Mudit Gupta, ${ }^{3}$ Sanyukta \\ Gurung \\ 'Department of Clinical Physiology, Maharajgunj Medical \\ Campus, Nepal \\ ${ }^{2}$ Department of General Practice and Emergency Medicine, \\ Maharajgunj Medical Campus, Nepal \\ ${ }^{3}$ Department of ENT - Head and Neck Surgery, Maharajgunj \\ Medical Campus, Nepal
}

Correspondence: Neeti Bhat, Lecturer, Madan Bhandari Academy of Health Sciences, Bgamati Province, Hetauda, Nepal, Tel+9779813286250, Email neetibhatbkt@gmail.com

Received: April 05, 2021 | Published: April 26, 2021
Abbreviations: AGE, advanced glycation end; dB, decibel; Ent, ear, nose and throat; GP, general practice; Hz, hertz; IOM, institute of medicine; IRC, institutional review committee; OPD, outpatient department; ROC, receiver operating characteristic; SD, standard deviation; WHO, World health organization

\section{Introduction}

Diabetes mellitus is a metabolic disorder characterized by chronic hyperglycemia. The underlying cause of diabetes could be due to insulin deficiency or tissue resistance to insulin or both. An altered biochemical profile produces advanced glycation end products (AGE), which is the primary underlying pathogenesis of diabetes mellitus. Macrovascular and microvascular complications make diabetes mell its multi-systemic in nature, also affecting the organ of hearing. ${ }^{1,2}$ Hearing is the basic means of communication and makes interpersonal interaction possible in daily lives. Hearing impairment leads to altered linguistic, cognitive, and social development. ${ }^{3}$ Hearing loss has a huge impact on the quality of life which is often neglected. Subjects with hearing loss face financial burden owing to loss of income and productivity. ${ }^{4}$ WHO defines a hearing threshold greater than 25 decibels in the better hearing ear as hearing loss. ${ }^{5}$

Realizing that we are confronted with huge burdens of diabetes mellitus, recently the Government of Nepal has started addressing diabetes mellitus on a policy level. The assessed pervasiveness of diabetes mellitus in Nepal is around 3.2\%, with a discernible rural-urban variation. ${ }^{6}$ The complications due to diabetes mellitus are on the rise which is mostly disabling or life-threatening. ${ }^{7}$ The common complications associated with diabetes mellitus are well appreciated with devised guidelines for its management, such as diabetic eye examination / renal function test. ${ }^{8}$ Auditory dysfunction is a complication of diabetes mellitus that is under-appreciated. ${ }^{9}$ The progressive, degenerative changes due to aging are found to be accelerated in diabetes mellitus patients. Thus, clinically the pattern of hearing loss in diabetes mellitus is similar to presbycusis; however, the onset of presbycusis is seen at an earlier age. Due to such overlapping features, the cause-and-effect relationship of diabetes mellitus and hearing loss has been debatable and the underlying mechanism of hearing loss in diabetes mellitus has not been clearly defined. ${ }^{10,11}$ So, the use of auditory tests to monitor hearing status in diabetic patients are neglected to date. Hence, our study aimed to find the prevalence of hearing loss in diabetes mellitus patients.

\section{Materials and methods}

Hospital-based obsevational cross-sectional study was conducted at the Tribhuvan University Teaching Hospital, Kathmandu Nepal. Department of Clinical Physiology, Department of General Practice and Emergency Medicine, and Department of ENT- Head and Neck Surgery collaborated to study the prevalence of hearing loss in diabetes mellitus patients. Ethical clearance and permission for conduction of the study were obtained from the Institutional Review Committee (IRC) of the Institute of Medicine (IOM) before the commencement of the study (Ref. no. 114 (6-11-E4) 075 /076).

The diabetes mellitus patients were selected from the outpatient department (OPD) of the Department of General Practice (GP) and Emergency medicine after meeting the inclusion criteria of having confirmed diagnosis by attending physician from September 2018 to November 2019. To rule out the influence of possible confounders on hearing, we excluded subjects with hypertension, history of 
consumption of ototoxic drugs, history of ear surgeries affecting hearing, history of ear infections, history of ear and head trauma. The hearing loss in diabetes mellitus is bilateral and usually symmetrical, progressive, and sensorineural..$^{12,13}$ So, we excluded patients evaluated in the ENT department who had asymmetrical or conductive hearing loss, audiograms suggestive of hearing loss due to Meniere's, otosclerosis, noise-induced hearing loss from the study. We explained details about the study to the study participants and obtained informed consent. Otolaryngologists conducted otoscope examination and tuning fork test and then the participants were then subjected to audiometric assessment to evaluate hearing status. Amplaid 137 plus or Amplaid 177 was used for audiological evaluation in a sound-treated room at Audiometry Lab of the department of ENT-Head and Neck Surgery. The test was conducted by an experienced audiologist after the audiometer was appropriately calibrated. The pure tone hearing threshold for both air conduction and bone conduction was analyzed in $250 \mathrm{Hertz}(\mathrm{Hz}), 500 \mathrm{~Hz}, 1000 \mathrm{~Hz}, 2000 \mathrm{~Hz}, 3000 \mathrm{~Hz}, 4000 \mathrm{~Hz}$, and $8000 \mathrm{~Hz}$. Any asymmetrical, non-bilateral, and non- sensorineural hearing loss in the audiogram was excluded. The average of pure tone threshold of $500 \mathrm{~Hz}, 1000 \mathrm{~Hz}, 2000 \mathrm{~Hz}$, and $4000 \mathrm{~Hz}$ of air conduction was calculated and considered pure tone average.

We considered the pure tone average of more than 25 decibels $(\mathrm{dB})$ in both ears as hearing loss. We then classified the hearing status as per the World Health Organization (WHO)guidelines. Pure tone average of 26-40 dB was graded as mild hearing loss, $41-60 \mathrm{~dB}$ as moderate hearing loss, $61-80 \mathrm{~dB}$ as a severe hearing loss, and $81 \mathrm{~dB}$ and above as a profound hearing loss. ${ }^{5,14}$ We used IBM SPSS Statistics for MacBook, version 23 (IBM Corp., Armonk, N.Y., USA) for statistical analysis. Continuous variables were expressed in mean $\pm \mathrm{SD}$. We used percentage and proportion to express categorical variables. Pearson's correlation was used to find the correlation between age and hearing threshold at different frequencies. The probability value ( $p$-value) less than 0.05 was considered to be significant. The receiver's operating characteristic curve was generated to find the best cut-off age for hearing loss in diabetics.

\section{Result}

The mean age of 55 diabetes mellitus patients recruited in the study was 52.1313 .31 years with the age range of $30-85$ years. In this study, the percentage of female patients was $58 \%$. Forty out of 55 patients in our study were of age group less than 60 years. There was an equal number of participants in the age group of 30-45 years and 46-60 years and fewer patients in the age group more than 60 years age group (Table 1).

Table I Demographic distribution of diabetic patients $(n=55)$

\begin{tabular}{lll}
\hline Age group & Male & Female \\
\hline $30-45$ & 7 & 13 \\
$46-60$ & 8 & 12 \\
$60+$ & 8 & 7
\end{tabular}

Our study participants had a normal cardiorespiratory profile with respect to systolic and diastolic blood pressure, heart rate, and respiratory rate (Table 2). The diabetes mellitus control was checked measuring the fasting and postprandial blood sugar before the patients were evaluated for the hearing status. The mean fasting and postprandial blood sugar were $159.70 \pm 58.66 \mathrm{mg} / \mathrm{dl}$ and $246.79 \pm 93.68$ $\mathrm{mg} / \mathrm{dl}$ respectively. We found that 17 diabetes mellitus patients had a hearing threshold greater than $25 \mathrm{~dB}$ in both ears. Thus, hearing loss was present in $30 \%$ of diabetes mellitus patients. Out of diabetes mellitus patients with hearing loss, 13 had hearing loss of mild grade and four had moderate hearing loss (Figure 1).

Table 2 Cardio-respiratory profile of diabetic patients $(n=55)$

\begin{tabular}{llll}
\hline Variables & Unit & Mean & SD \\
\hline $\begin{array}{l}\text { Systolic Blood } \\
\text { Pressure }\end{array}$ & $\mathrm{mmHg}$ & 120.89 & $8.6 \mathrm{I}$ \\
$\begin{array}{l}\text { Diastolic Blood } \\
\begin{array}{l}\text { Pressure } \\
\text { Respiratory Rate }\end{array}\end{array}$ & $\mathrm{mmHg}$ & 79.4 & 10.85 \\
$\begin{array}{l}\text { Per-minute } \\
\text { Heart Rate }\end{array}$ & Beats per minute & 72 & $1.5 \mathrm{I}$ \\
\hline
\end{tabular}

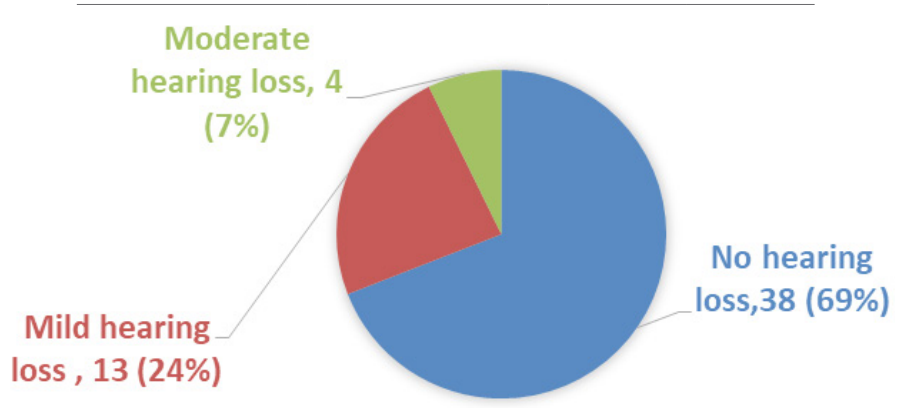

Figure I Hearing status in diabetic patients as per WHO hearing loss classification $(n=55)$.

The prevalence of hearing loss increased with an increase in frequency as more than half of participants had hearing loss at or above the frequency of $4000 \mathrm{Hertz}$ (Figure 2). At a frequency of 8000 $\mathrm{Hz}$, almost $75 \%$ of the patients had hearing loss of some degree while at $250-1000 \mathrm{~Hz}$ only about $22 \%$ had hearing loss.

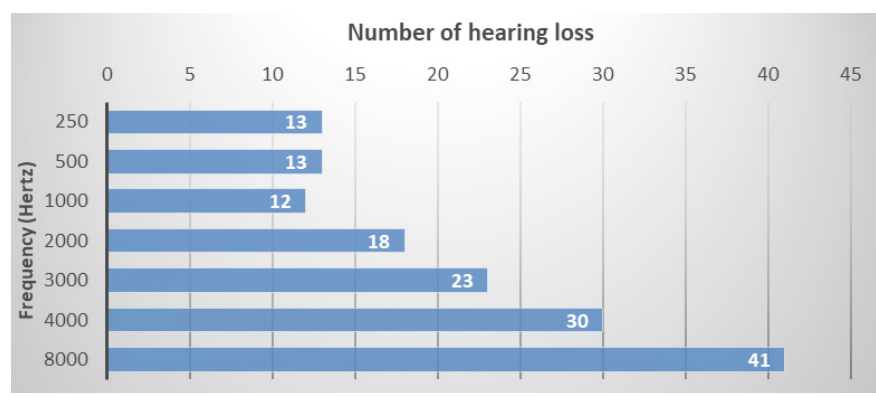

Figure 2 Prevalence of hearing loss at different frequencies in diabetes mellitus patients $(n=55)$.

In the correlation analysis between age and hearing threshold at different frequencies of diabetes mellitus patients, we found out that age significantly and positively correlated with hearing threshold across all frequencies tested i.e., the more the age higher the hearing threshold. The positive correlation coefficient was notably higher at higher frequencies i.e. greater the frequency higher the hearing threshold (Table 3). The receiver's operating characteristic (ROC) curve was generated to find a cut off age of hearing loss in diabetes mellitus study participants. The best cut-off years for the onset of hearing loss in diabetics was found to be 51.50 years with $81.3 \%$ sensitivity and $69.2 \%$ specificity (Figure 3 ). 
Table 3 Correlation of age with the threshold at different frequencies in diabetes mellitus patients $(n=55)$

\begin{tabular}{lll}
\hline $\begin{array}{l}\text { Frequencies } \\
\text { (Hertz) }\end{array}$ & $\begin{array}{l}\text { Correlation } \\
\text { coefficient }(\boldsymbol{r})\end{array}$ & p-value \\
\hline 250 & $0.313^{*}$ & 0.02 \\
500 & $0.335^{*}$ & 0.013 \\
1000 & $0.486^{*}$ & 0 \\
2000 & $0.434^{* *}$ & 0.001 \\
3000 & $0.514^{* *}$ & 0 \\
4000 & $0.562 * *$ & 0 \\
8000 & $0.689 * *$ & 0
\end{tabular}

*, Significant; **, highly significant

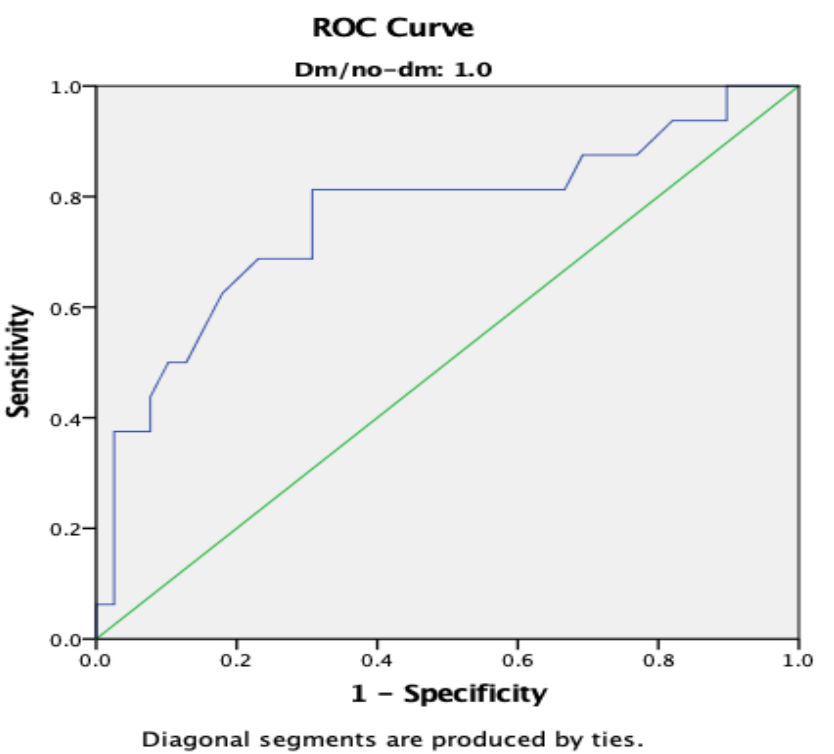

Figure 3 The receiver operating characteristic curve for the age of hearing loss in diabetes mellitus patients.

\section{Discussion}

In the current study, we found a high prevalence of hearing loss in diabetes mellitus and it was more prevalent in the elderly. We observed $30 \%$ of diabetes mellitus patients had sensorineural hearing loss of some degree. This finding was less compared to earlier studies where the prevalence of hearing loss in diabetes mellitus patients quoted from a range of $33.7 \%$ to $97.6 \% .{ }^{15-19}$ We did not find clarification for such a broad variation, but it could be attributed to the study methodology of differences in inclusion criteria, demographics, type and duration of diabetes mellitus, the clinical and biochemical status of diabetes mellitus patients. The current study did not limit the recruitment of study participants according to the duration of disease, age, or gender. The study by Khakurel et al., conducted at Kathmandu Medical College among type 2 diabetes mellitus patients observed hearing loss of $72.5 \%$. Nwosu et al., ${ }^{15}$ set inclusion criteria of minimum duration of disease for five years or more but did not have any age limits noted $46.9 \%$ prevalence of hearing loss in diabetes mellitus patients. But they did not exclude any subjects with conductive hearing loss. Among type 2 diabetes mellitus patients, Idugboe et al., ${ }^{16}$ observed the prevalence of hearing loss to be $71.4 \%$ where there was no prerequisite for a minimum duration of disease to be included in the study, and subjects with hearing loss of any type were enrolled in the study.

Systemic review and meta-analysis study to find an association between hearing loss and diabetes mellitus found out that the overall pooled odds of having a hearing impairment in diabetes mellitus patients was 1.91- 2.15 when compared with non-diabetes Mellitus healthy subjects. ${ }^{20,21}$ In the present study, the mean age of study participants was $52.13 \pm 13.31$ years which was similar to earlier studies. ${ }^{15-17}$ Similarly, female predominance in the current study was in line with existing studies. ${ }^{20-22}$ Gyawali et al. ${ }^{24}$ observed that type 2 diabetes mellitus exhibited pronounced excess of females in the Nepalese population.

When hearing status was categorized as per WHO guidelines, most diabetes mellitus patients with hearing loss belonged to mild grade hearing loss. Hence, the hearing loss associated with diabetes mellitus was mostly subclinical, which is why it is mostly ignored by subjects. Our finding was in concordance with previous studies conducted in India. ${ }^{18,25}$ Dosemane et al. ${ }^{17}$ asserted that there were far lesser complaints of ear problems (39\%) by diabetes mellitus patients when contested to the actual prevalence $(97.2 \%$ ) in terms of audiological evaluation in their South Indian study population. Corroborating to Dosemane et al., ${ }^{17}$ Soares et al., ${ }^{26}$ observed that the prevalence of hearing loss in diabetes mellitus patients was quite low (2.56\%) when it was self-reported. We observed that age significantly correlated positively with hearing threshold throughout all frequencies tested. The correlation coefficient increased with an increase in frequency. The higher prevalence of hearing loss at higher frequencies also substantiated previous findings of higher frequencies affected more in diabetes mellitus. ${ }^{15,16}$ Akinpelu et al., ${ }^{27}$ examined histopathological findings in the cochlear structure of diabetes mellitus patients, where they noted changes in stria vascularis and Organ of Corti. The author also reported atrophy of spiral ganglion more at basal turn of the cochlea. Higher frequency is detected at the basal turn, which is why higher frequencies were found to be more affected in our diabetes mellitus patients.

Our observation did not completely replicate observations by Meena et al., ${ }^{28}$ who indicated a positive correlation between diabetes mellitus and hearing threshold only at $750 \mathrm{~Hz}$ and $1000 \mathrm{~Hz}$. Their study population was below 50 years and the author did not surmise the finding. To corroborate with existing studies that have insisted that diabetes mellitus speeds up Presbycusis, we further explored to find out the best cut off age of onset of hearing loss in diabetes mellitus patients. To perform this, we entailed Receiver Operators Curves and found 51.50 50 years as the best cut-off age for onset of hearing loss in diabetes mellitus patients which suggests the premature onset of hearing loss in diabetes mellitus patients. ${ }^{29}$ Some limitations of the current study are the research methodology of observational crosssectional study which comes with inherent shortcomings of temporal weakness and inability to control confounders. There was an absence of data on different attributes of diabetes mellitus such as duration of disease, glycaemic index, medications that could have affected the disease process.

To conduct any further study, a population-based survey longitudinal study with a more rigorous methodology to see the effect of diabetes mellitus on hearing status to identify the cause and answer complexity of pathogenesis is recommended. Also, future research could include different attributes of diabetes and its effect on the hearing threshold. 


\section{Conclusion}

We observed a considerable number of diabetes mellitus patients had hearing loss with a prevalence of $30 \%$. The elderly were more affected by hearing loss predominantly at higher frequencies.

\section{Acknowledgments}

We would like to thank Dr. Dinesh Banstola, Prof Dr. Bibhu Pradhan, and Dr. Yogendra Shakya for allowing us to conduct research. Also, we acknowledge Mr. Jitendra Shrivastav, Audiologist, and Mr. Bibek Poudel of Audiology Lab where all the audiological tests were performed.

\section{Conflicts of interest}

Authors declare that there is no conflict of interest.

\section{Funding}

None.

\section{References}

1. Kharroubi AT, Darwish HM. Diabetes Mellitus: The Epidemic of The Century. World J Diabetes. 2015;25;6(6):850-867.

2. Singh VP, Bali A, Singh N, et al. Advanced Glycation End Products and Diabetic Complications. Korean J Physiol Pharmacol Off J Korean Physiol Soc Korean Soc Pharmacol. 2014;18(1):1-14

3. Brownell WE. How The Ear Works - Nature's Solutions For Listening. Volta Rev. 1997;99(5):9-28.

4. Huddle MG, Goman AM, Kernizan FC, et al. The Economic Impact of Adult Hearing Loss: A Systematic Review. JAMA Otolaryngol Neck Surg. 2017;143(10):1040-1048

5. WHO. Global Estimates on Prevalence of Hearing Loss. 2012.

6. Upreti SR, Lohani GR, Magtymova A, et al. Strengthening Policy and Governance To Address The Growing Burden of Diabetes in Nepal. WHO South-East Asia J Public Health. 2016;5(1):40.

7. King G, Yu MG. Harvard Health Blog - Harvard Health Publishing. Why Are Diabetes-Related Complications on The Rise? 2019.

8. Sapra A, Bhandari P. Diabetes Mellitus. In: StatPearls. Treasure Island (FL): StatPearls Publishing. 2020.

9. Morrison CL, Morar P, Morrison G, et al. Hearing Loss and Type 2 Diabetes: Is There a Link? Pract Diabetes. 2014;31(9):366-369.

10. Oh I-H, Lee JH, Park DC, et al. Hearing Loss as a Function of Aging and Diabetes Mellitus: A Cross Sectional Study. PLoS ONE. 2014;9(12).

11. Krishnappa S, Naseeruddin K. A Clinical Study of Age Related Hearing Loss Among Diabetes Patients. Indian J Otol. 2014;20(4):160.

12. Srinivas CV, Shyamala V, Shiva Kumar BR. Clinical Study to Evaluate the Association Between Sensorineural Hearing Loss and Diabetes Mellitus in Poorly Controlled Patients Whose HbAlc $>8$. Indian J Otolaryngol Head Neck Surg. 201668(2):191-195.
13. Misra V, Agarwal CG, Bhatia N, et al. Sensorineural Deafness in Patients of Type 2 Diabetes Mellitus in Uttar Pradesh: A Pilot Study. Indian J Otolaryngol Head Neck Surg. 2013;65(Suppl 3):532-536.

14. WHO. Grades of hearing impairment. WHO. World Health Organization.

15. Nwosu JN, Chime EN. Hearing Thresholds in Adult Nigerians With Diabetes Mellitus: A Case-Control Study. Diabetes Metab Syndr Obes Targets Ther. 2017;10:155-160.

16. Idugboe OJ, Kolawole BA, Totyen EL. Hearing Threshold Level among Adult Diabetics in South-Western Nigeria. $J$ Otolaryngol Rhinol. 2018;4(2).

17. Dosemane D, Bahniwal RK, Manisha N, et al. Association Between Type 2 Diabetes Mellitus and Hearing Loss Among Patients in a Coastal City of South India. Indian J Otolaryngol Head Neck Surg. 2019;71(Suppl 2):1422-1425.

18. Sachdeva K, Azim S. Sensorineural Hearing Loss and Type II Diabetes Mellitus. Int J Otorhinolaryngol Head Neck Surg. 2018;4(2):499-507.

19. Khakurel G, Mahato NB. Evaluation of Hearing loss by Pure Tone Audiometry in Type 2 Diabetes Mellitus. Nepal Med J. 2020 ;3(1):286289.

20. Akinpelu OV, Mujica-Mota M, Daniel SJ. Is Type 2 Diabetes Mellitus Associated with Alterations in Hearing? A Systematic Review and MetaAnalysis. Laryngoscope. 2014;124(3):767-776.

21. Horikawa C, Kodama S, Tanak S, et al. Diabetes and Risk of Hearing Impairment in Adults: A Meta-Analysis. J Clin Endocrinol Metab. 2013;98(1):51-58.

22. Hosseini MS, Saeedi M, Khalkhali SA. Prevalence of Hearing Disorders among Type 2 Diabetes Mellitus Patients with and without Vitamin D Deficiency. Mcedica. 2020;15(1):32-36.

23. Ren $\mathrm{H}$, Wang Z, Mao Z, et al. Hearing Loss in Type 2 Diabetes in Association with Diabetic Neuropathy. Arch Med Res. 2017;48(7):631637.

24. Gyawali B, Sharma R, Neupane D, et al. Prevalence of type 2 diabetes in Nepal: a systematic review and meta-analysis from 2000 to 2014. Glob Health Action. 2015;26;8.

25. Aziz A, Shetty K, Bhat VS. Clinical Evaluation of Sensorineural Hearing Loss in Diabetes Mellitus. IOSR J Dent Med Sci. 2016;15(1).

26. Soares M, Oenning N, Ziegelmann P, et al. Association Between SelfReported Hearing Impairment and Diabetes: A Brazilian PopulationBased Study. Arch Public Health. 2018;76(1):62.

27. Akinpelu OV, Ibrahim F, Waissbluth S, et al. Histopathologic Changes in The Cochlea Associated with Diabetes Mellitus--A Review. Otol Neurotol Off Publ Am Otol Soc Am Neurotol Soc Eur Acad Otol Neurotol. 2014;35(5):764-74.

28. Meena R, Sonkhya D, Sonkhya N. Evaluation of Hearing Loss in Patients with Type 2 Diabetes Mellitus. Int J Res Med Sci. 2017;4(6):2281-2287.

29. Wang J, Puel J-L. Presbycusis: An Update on Cochlear Mechanisms and Therapies. J Clin Med. 2020;14;9(1). 